\title{
Astrophysical bounds on global strings
}

\author{
Shane L. Larson* and William A. Hiscock ${ }^{\dagger}$ \\ Department of Physics, Montana State University, Bozeman, Montana 59717
}

(Received 9 April 1997)

\begin{abstract}
Global topological defects produce nonzero stress energy throughout spacetime, and as a result can have observable gravitational influence on surrounding matter. Gravitational effects of global strings are used to place bounds on their cosmic abundance. The minimum separation between global strings is estimated by considering the defects' contribution to the cosmological energy density. More rigorous constraints on the abundance of global strings are constructed by examining the tidal forces such defects will have on observable astrophysical systems. The small number of observed tidally disrupted systems indicates there can be very few of these objects in the observable Universe. [S0556-2821(97)04318-X]

PACS number(s): 98.80.Cq, 11.27.+d
\end{abstract}

\section{INTRODUCTION}

Topological defects in spacetime arise from the spontaneous breaking of local and global gauge symmetries. Such defects may have been created during vacuum phase transitions in the early Universe, and could have interesting cosmological implications, particularly in the production of density fluctuations leading to the formation of large scale structures [1,2]. Although recent simulations suggest that neither gauge topological defects [3] nor global defects [4] are likely to be the dominant mechanism for structure formation, the bounds derived on global string abundance in this paper are quite general and cover a wide range of defect energy scales, including scales much lower than those typically associated with structure formation.

Defects associated with local symmetry breaking, such as gauged (magnetic) monopoles and gauged cosmic strings, are known to have no substantial stress energy associated with the fields outside the core of the defect. As a result, the spacetime exterior to the defect is a vacuum solution of the Einstein equations $[5,6]$, and, in the case of the gauged cosmic string, it is actually flat $[7,8]$. Individual gauged monopoles have insignificant gravitational effects, while gauged cosmic strings are influential only when radiation or matter passes on opposite sides of the string (e.g., gravitational lensing). Domain walls have large gravitational effects [9], which may be used to strongly constrain their existence in the early Universe.

Global defects differ from gauge defects in that there is no gauge field associated with the symmetry breaking. As a result, the scalar fields vary spatially throughout the spacetime, yielding a nonzero stress-energy tensor that can lead to significant gravitational effects [10-12].

Previous work [13] has shown that the gravitational fields associated with a single global monopole can be used to constrain the number density of such objects in the observable Universe. In this paper, similar considerations are applied to the spacetime of a global string to develop astrophysical constraints that will provide upper bounds to the

\footnotetext{
*Electronic address: shane@ orion.physics.montana.edu

†Electronic address: billh@orion.physics.montana.edu
}

abundance of such defects. While the constraints developed here are based on the spacetime metric of an infinitely long, cylindrically symmetric global string, they may also be applied to string loops, as long as the distance to the string is less than the local radius of curvature of the loop.

Topological defects such as global strings can be created as a result of phase transitions in the early Universe. After the phase transition, the strings will evolve in a network towards an asymptotic state with some number of long strings per cosmological horizon volume, along with a spectrum of different sized loops of string. Such networks have been studied extensively for gauge strings [14], but much less attention has been paid to the case of global strings [15]. The limits derived here are too weak to seriously constrain any of the proposed network models; on the other hand, these limits are only dependent on the local physics of an existing global string, and not on any assumptions about its genesis or subsequent network evolution.

In Sec. II the exact metric describing a static, cylindrically symmetric global string [11] is reviewed and an approximation to the metric is developed that will allow the constraints to be stated in analytic form.

In Sec. III bounds are constructed based on the strings' contribution to the total cosmological energy density. Based on an extremely simple model, the average distance between global strings today must exceed $30\left[\eta /\left(10^{16} \mathrm{GeV}\right)\right] \mathrm{Mpc}$, where $\eta$ is the energy scale of the strings.

In Sec. IV somewhat more general bounds are developed by examining the possible tidal disruption of various astrophysical systems by the gravitational field of a global string. A general expression is developed for the tidal disruption distance $d$, which is the closest a system bound by gravity can approach a global string without being tidally disrupted by the string's spacetime curvature. Several example constraints are developed using this expression. In order to prevent global strings from tidally disrupting an unacceptable fraction of all galaxies, it is shown that strings must be separated by a distance exceeding $3\left[\eta /\left(10^{16} \mathrm{GeV}\right)\right] \mathrm{Mpc}$. A bound at lower string energy scales may be obtained by considering the Earth-Moon system. Lunar laser ranging data assures that no global string with an energy scale $\eta>7 \times 10^{8}$ $\mathrm{GeV}$ has passed within 5 times the distance between the Earth and Moon within the past 28 years. 
Sign conventions and notation follow those of Misner, Thorne, and Wheeler [16]; natural units $(G=c=\hbar=1)$ are used throughout.

\section{SPACETIME OF A GLOBAL STRING}

The simplest example of a scalar field Lagrangian density for which global strings are a possible consequence of symmetry breaking is a single complex scalar field with a global $\mathrm{U}(1)$ symmetry [1]:

$$
\mathcal{L}=\partial_{\mu} \phi^{\dagger} \partial^{\mu} \phi-\frac{1}{2} \lambda\left(\phi^{\dagger} \phi-\eta^{2}\right)^{2} .
$$

This Lagrangian is invariant under the global gauge transformation

$$
\phi \rightarrow \widetilde{\phi}=e^{i \Lambda} \phi
$$

where $\Lambda$ is an arbitrary constant. A global string solution has the general form

$$
\phi=F(r) e^{i \theta},
$$

with $F(0)=0$ and $F(r)=\eta$ for all $r \geqslant r_{C}$, where $r_{C}$ is the core radius of the string, roughly given by $r_{C} \approx 1 / \eta$. In a typical grand unified theory, the parameter $\eta$ might be of order $10^{16} \mathrm{GeV}$.

Cohen and Kaplan [11] have found the exact spacetime metric for a static, cylindrically symmetric global string such as that described above. The metric is

$$
d s^{2}=\left(\frac{u}{u_{0}}\right)\left(-d t^{2}+d z^{2}\right)+A(u)\left(d u^{2}+d \theta^{2}\right),
$$

where

$$
A(u)=\frac{1}{\eta^{2}} \sqrt{\frac{u_{0}}{u}} \exp \left[\left(u_{0}^{2}-u^{2}\right) / u_{0}\right]
$$

and

$$
u_{0}=\frac{1}{8 \pi \eta^{2}}
$$

Here $u$ is a dimensionless radial coordinate that decreases outward from the string. The axis of cylindrical symmetry is located at $u=\infty$, the surface of the string is at $u \simeq u_{0}$, and there is a timelike curvature singularity at $u=0$, which is at a finite proper spatial distance from the core. The metric given in Eq. (4) is valid outside the core region, $u \lesssim u_{0}$.

Cohen and Kaplan have derived an elementary upper bound on the energy scale of the global string by requiring that the distance from the core to the outer singularity be greater than the size of the present cosmological horizon. This places an upper limit of $\eta \lesssim 2.0 \times 10^{17} \mathrm{GeV}$ on the energy scale of the string.

The nature of the dimensionless radial coordinate $u$ makes intuition about the global string system difficult. In order to gain further insight into the global string spacetime, it is desirable to devise a method by which proper radial distances from the string can be obtained for given values of $u$. One approach is to directly calculate the proper radial distance from the metric, integrating along a curve of fixed $t$, $\theta$, and $z$ from the surface of the string at $u_{0}$ to the desired location at $u$ :

$$
\begin{aligned}
r_{p} & =\int d s=\int_{u}^{u_{0}} \sqrt{g_{u u}} d u \\
& =\int_{u}^{u_{0}} \frac{1}{\eta}\left(\frac{u_{0}}{u}\right)^{1 / 4} \exp \left[\frac{u_{0}^{2}-u^{2}}{2 u_{0}}\right] d u .
\end{aligned}
$$

This has the disadvantage that it does not appear to be possible to evaluate the integral in terms of simple functions. A much simpler method is to use the cylindrical symmetry of the system to advantage by defining a circumferential radius (analogous to the usual area or curvature coordinate $r$ in the Schwarzschild metric) by $r_{c}{ }^{2}=g_{\theta \theta}$, or

$$
r_{c}=\sqrt{g_{\theta \theta}}=\frac{1}{\eta}\left(\frac{u_{0}}{u}\right)^{1 / 4} \exp \left[\frac{u_{0}^{2}-u^{2}}{2 u_{0}}\right] \text {. }
$$

One may use these expressions for $r_{p}$ and $r_{c}$ to determine what values of $u$ correspond to particular distances for a variety of energy scales, $\eta$, for the string. Astrophysically relevant distance scales turn out to generally correspond to values of $u$ close to $u_{0}$.

An approximation scheme can be developed to simplify computations in this spacetime by expanding the metric functions in power series for values of $u$ close to $u_{0}$. Defining a new coordinate $\delta$ by

$$
\delta=\frac{u_{0}-u}{u_{0}}
$$

the metric, rewritten in terms of the new coordinate and expanded to first order in $\delta$, becomes

$$
d s^{2}=(1-\delta)\left(-d t^{2}+d z^{2}\right)+\frac{1}{\eta^{2}} e^{2 u_{0} \delta}\left(u_{0}^{2} d \delta^{2}+d \theta^{2}\right) .
$$

At first glance, one might object to this form of the metric on the grounds that the approximation does not appear to have been carried out to completion. The appearance of the exponential function containing the small parameter $\delta$ suggests that the exponential should be expanded to first order in $\delta$. This is not possible because the argument of the exponential is $u_{0} \delta$, which in the domains of physical interest typically has values of order $10^{2}$. This approximation is similar, but not identical to, the linearized global string metric discussed in Refs. [11,17].

Using this approximation the proper and circumferential radii may be simply expressed in terms of the coordinate $\delta$ as

$$
r_{p}=r_{c}=\frac{e^{u_{0} \delta}}{\eta} \equiv r
$$

where the last equality indicates that a new coordinate $r$ is defined by this equation, within the context of the approximate metric.

The approximate metric then takes on a particularly simple form when expressed in terms of the coordinate $r$, 


$$
d s^{2} \approx\left[1-\frac{1}{u_{0}} \ln (r \eta)\right]\left(-d t^{2}+d \eta^{2}\right)+d r^{2}+r^{2} d \theta^{2} .
$$

Written in this form, our approximate metric is seen to be equivalent to the linearized metric $[11,17]$, with the further simplification of ignoring terms of order $1 / u_{0}$ in $g_{\theta \theta}$, but not in $g_{t t}$ and $g_{z z}$. In every case examined, we have checked the validity of the results by comparison with numerical integration using the full metric of Eq. (4). Results will generally be stated in terms of the approximate coordinate $r$, as this allows simple analytic forms for the constraints and simple interpretation of the radial coordinate.

\section{COSMOLOGICAL DENSITY CONSTRAINTS}

Global strings differ from cosmic strings in that they have nonconstant scalar fields extending throughout the spacetime, and hence will have significant gravitational influence on observable astrophysical systems. This fact can be used to construct constraints on the abundance of global strings at the current epoch.

The scalar fields associated with a global string will contribute to the total energy density of the spacetime. An average energy density due to these scalar fields, $\bar{\rho}$, within a cylindrical volume, can be estimated by dividing the integrated mass in the scalar field by the cylindrical volume of the spacetime out to fixed radius $r$. A number of different estimates may be constructed, depending upon how one imagines the global strings filling spacetime within a cosmological model. The simplest possible model is examined here, assuming the Universe to be filled with a network of parallel global strings and treating each string as if it is in flat space [appropriate to the approximate metric of Eq. (12)]. The energy density in the scalar fields is approximately given by

$$
\rho \sim \frac{\eta^{2}}{r^{2}}
$$

If the typical distance between strings is defined to be $2 r_{0}$, then the mass contributed by the scalar fields within the radius $r_{0}$, per unit length, is

$$
M \sim 2 \pi \int_{0}^{r_{0}} \frac{\eta^{2}}{r^{2}} r d r \sim 2 \pi \eta^{2} \ln \left(r_{0} \eta\right) .
$$

This mass occupies the spatial volume per unit length of string

$$
V \sim \pi r_{0}^{2},
$$

leading to an average mass density given by

$$
\bar{\rho} \sim \frac{\eta^{2}}{r_{0}^{2}} \ln \left(r_{0} \eta\right) .
$$

A constraint on the abundance of global strings may be obtained from this result by insisting that $\bar{\rho}$ be less than ten times the closure density of the Universe, a rough upper limit on the total cosmological density of matter consistent with present astronomical observations. The separation for all energy scales $\eta$ of potential interest is such that the logarithmic term in Eq. (16) will always be of order $10^{2}$. This implies a minimum separation of

$$
r_{0} \geqslant 7 \times 10^{26} h^{-1}\left(\frac{\eta}{10^{16} \mathrm{GeV}}\right) \mathrm{cm} \approx 20 h^{-1}\left(\frac{\eta}{10^{16} \mathrm{GeV}}\right) \mathrm{Mpc},
$$

where $h$ is the Hubble constant in units of $100 \mathrm{~km} \mathrm{sec}^{-1} \mathrm{Mpc}^{-1}$. This limit depends rather strongly on the model used for the string distribution, the assumption that all strings are infinite in length (no loops), etc. More rigorous constraints can be placed on global string abundances by considering limits that do not depend on cosmological assumptions, but are instead determined by local astrophysical effects of a single string.

\section{TIDAL ACCELERATION CONSTRAINTS}

The stress energy of the global scalar fields associated with the string will give rise to nonzero curvature of the spacetime. Extended astrophysical systems will experience tidal accelerations due to the string; these accelerations may be examined by using the equation of geodesic deviation. Given an extended body whose center of mass follows a geodesic with four-velocity $u^{\alpha}$, the acceleration of a point in the body relative to the center of mass is given by

$$
a^{\mu}=-R_{\alpha \beta \nu}^{\mu} u^{\alpha} n^{\beta} u^{\nu},
$$

where $n^{\alpha}$ is the body vector orthogonal to $u^{\alpha}$ connecting the point to the center of mass, and $R^{\mu}{ }_{\alpha \beta \nu}$ is the Riemann tensor of the background (global string) spacetime.

Astrophysical systems such as the solar system, the galaxy, etc. are bound by gravity. Such systems may be tidally disrupted, "ionized" in a sense, if they should approach a global string too closely. By examining the tidal accelerations $\left(a_{T}\right)$ caused by a global string using Eq. (18) and comparing them to the Newtonian gravitational accelerations binding the system together $\left(a_{N}\right)$, a tidal disruption distance $d$ may be computed such that at the distance $d$,

$$
a_{T}=a_{N} .
$$

If a system passes closer to a global string than $d$, it will be tidally disrupted.

It is convenient to compute the Riemann tensor components in an orthonormal frame; this simplifies the later calculation of the components of the tidal acceleration in the frame attached to a freely falling observer. The basis oneforms for an orthonormal frame, which is static with respect to the string, may be expressed as

$$
\begin{gathered}
\omega^{\hat{0}}=\left(\frac{u}{u_{0}}\right)^{1 / 2} d t \approx\left(1-\frac{1}{2 u_{0}} \ln (r \eta)\right) d t, \\
\omega^{\hat{3}}=\left(\frac{u}{u_{0}}\right)^{1 / 2} d z \approx\left(1-\frac{1}{2 u_{0}} \ln (r \eta)\right) d z, \\
\omega^{\hat{1}}=A(u)^{1 / 2} d u \approx d r,
\end{gathered}
$$


and

$$
\omega^{\hat{2}}=A(u)^{1 / 2} d \theta \approx r d \theta
$$

In such a frame, the components of the Riemann tensor can be written as

$$
\begin{aligned}
& R^{\hat{2} \hat{0}} \hat{2} \hat{0}=\frac{1}{A(u)}\left[\frac{1}{8 u^{2}}+\frac{1}{2 u_{0}}\right] \approx \frac{1}{2 u_{0} r^{2}}, \\
& R^{\hat{2} \hat{1}_{\hat{2}} \hat{1}}=\frac{1}{2 A(u)}\left[\frac{2}{u_{0}}-\frac{1}{2 u^{2}}\right] \approx \frac{1}{u_{0} r^{2}}, \\
& R^{\hat{3} \hat{0}} \hat{30}=-\frac{1}{4 A(u)} \frac{1}{u^{2}} \approx \frac{-1}{4 u_{0}{ }^{2} r^{2}}, \\
& R^{\hat{3} \hat{1}} \hat{\hat{1}} \hat{1}=\frac{1}{A(u)}\left[\frac{1}{8 u^{2}}-\frac{1}{2 u_{0}}\right] \approx \frac{-1}{2 u_{0} r^{2}}, \\
& R^{\hat{0} \hat{1}} \hat{0} \hat{1}=\frac{1}{A(u)}\left[\frac{1}{8 u^{2}}-\frac{1}{2 u_{0}}\right] \approx \frac{-1}{2 u_{0} r^{2}},
\end{aligned}
$$

where only the lowest order terms in $1 / u_{0}$ have been kept in the approximate expressions. The components of the tidal acceleration felt by an extended body are given by Eq. (18), and are computed in an orthonormal frame attached to a radially freely falling observer. The four-velocity of such an observer is

$$
\begin{gathered}
u^{\hat{0}}=\gamma, \\
u^{\hat{1}}=\gamma v,
\end{gathered}
$$

where $\gamma=1 / \sqrt{1-v^{2}}$, and $v$ is the radial three-velocity measured by a static observer. The freely falling frame is related to the static orthonormal frame by a simple Lorentz transformation. The orthonormal basis vectors of the freely falling frame, $e_{\tilde{\alpha}}$, have components in the static orthonormal frame given by

$$
e_{0}{ }_{0}^{\hat{\alpha}}=u^{\hat{\alpha}}, \quad e_{1}^{\tau^{\hat{\alpha}}}=n^{\hat{\alpha}}, \quad e_{2}^{\tau^{\hat{\alpha}}}=\delta_{2}{ }^{\hat{\alpha}}, \quad e_{3}^{{ }_{3}^{\hat{\alpha}}}=\delta_{3}{ }^{\hat{\alpha}},
$$

where $n^{\hat{\alpha}}$ is the unit length spacelike vector in the $\hat{0}, \hat{1}$ [or $(t, u)]$ plane orthogonal to $u^{\alpha}$. Computing the components of the tidal accelerations in the freely falling frame then yields

$$
\begin{aligned}
& a_{\tilde{1}}=\ell_{\tilde{1}} R^{\hat{0} \hat{1}} \hat{0} \hat{1},
\end{aligned}
$$

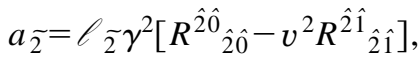

$$
\begin{aligned}
& a_{\tilde{3}}=\ell_{\tilde{3}} \gamma^{2}\left[R^{\hat{3} \hat{0}} \hat{30}-v^{2} R^{\hat{3} \hat{1}} \hat{\hat{1}} \hat{1}\right],
\end{aligned}
$$

where $\rho^{\tilde{\alpha}}$ is the body vector of the system. Substituting the approximate values for the Riemann tensor components from Eqs. (24)-(28),

$$
a_{\tilde{1}} \approx \frac{-\ell_{\tilde{1}}}{2 u_{0} r^{2}},
$$

$$
\begin{gathered}
a_{\tilde{2}} \approx \frac{\ell_{\tilde{2}} \gamma^{2}}{2 u_{0} r^{2}}\left(1-2 v^{2}\right), \\
a_{\widetilde{3}} \approx \frac{\ell_{\tilde{3}} \gamma^{2}}{4 u_{0}{ }^{2} r^{2}}\left(2 u_{0} v^{2}-1\right) .
\end{gathered}
$$

The maximum tidal acceleration on an extended system depends on the orientation of its body vectors and its velocity relative to the string. Global strings are, from a Newtonian viewpoint, gravitationally repulsive [11]; as seen by static observers, a body falling inward on a radial geodesic slows, reaches a turning point at a minimum radius, and then accelerates (in a coordinate sense) outward from the string. Since the tidal accelerations depend inversely on the distance to the string, but directly on the velocity relative to the string, it is not clear a priori where the tidal accelerations will be maximized. Examination of the tidal accelerations for the exact Riemann tensor and metric shows that the tidal accelerations diverge as $u \rightarrow 0$, as expected, as that is the curvature singularity surrounding the string. Once away from the curvature singularity, the tidal accelerations pass through a local maximum at the point of closest approach to the string for the cases of interest. The strongest constraints on astrophysical distances to global strings will therefore be obtained by setting $v=0, \gamma=1$ in Eqs. (35)-(37). Examination of Eqs. (35)-(37) shows that the tidal accelerations will generically be of order

$$
a_{T} \approx \frac{\ell}{2 u_{0} r^{2}} .
$$

If we then set this acceleration equal to the Newtonian acceleration binding a system of size $\ell$ and mass $M$ together, the critical tidal disruption distance is [using Eq. (6)]:

$$
d \simeq 2 \sqrt{\pi} \eta \frac{\ell^{3 / 2}}{M^{1 / 2}}
$$

This approximate equation has been compared with exact results obtained by numerical integration of the geodesic deviation equation, utilizing the exact metric of Eq. (4). The approximation is found to be remarkably good for all astrophysical systems of interest, agreeing with the exact results to about one-tenth of a percent or better. Above $10^{17} \mathrm{GeV}$, the approximation breaks down due to the proximity of the curvature singularity at $u=0$, which moves inward as the energy scale is increased.

The expression of Eq. (39) may be used to construct constraints on the allowable distances between strings (lest all astrophysical systems be disrupted) or between specific systems and the nearest global string. In the remainder of this section, the tidal disruption distance is evaluated for several systems of interest, and some basic constraints on how plentiful global strings may be are thereby developed.

The expression for the tidal disruption distance in Eq. (39) is evaluated for several systems of interest below, with conventional units restored: 


$$
\begin{gathered}
\text { Earth-Moon: } \quad d \simeq 850\left(\frac{\eta}{10^{16} \mathrm{GeV}}\right) \ell_{\text {Earth-Moon }}=3.3 \times 10^{8}\left(\frac{\eta}{10^{16} \mathrm{GeV}}\right) \mathrm{km}, \\
\text { Sun-Pluto: } \quad d \simeq 180\left(\frac{\eta}{10^{16} \mathrm{GeV}}\right) \ell_{\text {Pluto }}=7300\left(\frac{\eta}{10^{16} \mathrm{GeV}}\right) \mathrm{AU}, \\
\text { Galaxy: } \quad d \simeq 5.2\left(\frac{\eta}{10^{16} \mathrm{GeV}}\right) \ell_{\text {galaxy }} \simeq 150\left(\frac{\eta}{10^{16} \mathrm{GeV}}\right) \mathrm{kpc}, \\
\text { Cluster of Galaxies: } \quad d \simeq 5.2\left(\frac{\eta}{10^{16} \mathrm{GeV}}\right) \ell_{\text {cluster }} \simeq 15\left(\frac{\eta}{10^{16} \mathrm{GeV}}\right) \mathrm{Mpc},
\end{gathered}
$$

where $\ell_{\text {Earth-Moon }}$ and $\ell_{\text {Pluto }}$ are the semimajor axes of the orbits of those systems, and $\ell_{\text {galaxy }}$ and $\ell_{\text {cluster }}$ are the "typical" radii of those systems. These constraints, and similar bounds constructed for other systems, may be used to place bounds on the distance between global strings, or between the Earth and the nearest string.

The use of the equation of geodesic deviation is only valid if the extended body is small compared to its separation from the string. The critical distances listed above based on disruption of a galaxy or a cluster are then only valid if the string energy scale is $\gtrsim 10^{16} \mathrm{GeV}$; for the smaller systems (Earth-Moon and Sun-Pluto), the derivation is valid for an order of magnitude or so smaller energy. If one considers a system that experiences tidal perturbations smaller than total disruption, then the critical distance is increased, and the minimum string energy constrained is reduced.

As an example, consider the Earth-Moon system. The Moon's orbit has been studied for centuries, with steadily increasing precision, until in the last 28 years lunar laser ranging [18] has reduced the uncertainty in the Earth-Moon distance to $<3 \mathrm{~cm}$. If one assumes that the Earth-Moon system has not suffered an unexplained acceleration (perhaps due to a passing global string) sufficient to cause a $3 \mathrm{~cm}$ change in the Earth-Moon distance, then this implies that the global string tidal acceleration is bounded above by

$$
a_{T} \lesssim \frac{M}{\ell_{\text {Earth-Moon }}^{2}}-\frac{M}{\left(\ell_{\text {Earth-Moon }}+3 \mathrm{~cm}\right)^{2}} \approx 1.6 \times 10^{-10} a_{N} .
$$

The distance $d$ within which no string must have passed, lest it create such a perturbing acceleration, may then be evaluated from Eq. (44) using Eq. (38) for the tidal acceleration

$$
d_{l l r} \gtrsim 0.84\left(\frac{\eta}{10^{16} \mathrm{GeV}}\right) \mathrm{pc} .
$$

One can then state that, on the basis of lunar laser ranging, no global string with an energy scale of $10^{16} \mathrm{GeV}$ has passed within a parsec of the Earth-Moon system within the last 28 years. Alternately, examining the lower-energy limit of this bound, if we demand that $d>5 \ell$ for the geodesic deviation equation to be valid, then Eq. (45) implies that no global string with an energy scale $\eta>7 \times 10^{8} \mathrm{GeV}$ has passed within 5 times the distance between the Earth and Moon within the past 28 years.
One might guess that it would be easy to detect strings in such close proximity by observing the gravitational lensing caused by the string in the astrometry of nearby stars. Although the large number of stars available to be lensed greatly increases the likelihood of a lensing event with a nearby string, determining if galactic stars have been lensed is difficult at best. Determining if any two stars in an astrometric survey are actually lensed images of each other will pose a great challenge unless detailed spectrographic data exist for each star in the survey. Alternately, instead of using spectroscopy to identify paired images, one could use a time series of sensitive astrometric measurements to search for image motions that would be due to string motion rather than simple stellar proper motions.

Extensive astrometry at the milliarcsecond scale does exist for about $10^{5}$ stars in the recently published Hipparcos catalog. To gain an idea of whether string-induced aberrations of stellar positions would be detectable in this catalog, one can construct an estimate of the total number of stars in the catalog lensed by a nearby string. Lensing due to global strings has been shown to be approximately the same as the lensing produced by ordinary gauge cosmic strings $[11,17,19]$. The conical space of a gauge string has a deficit angle $\Delta \sim 8 \pi \eta^{2}$. We approximate the lensing of a global string by saying that the string will lens any star in a ribbon on the sky surrounding the string of width $\Delta$. The Hipparcos catalog has an average stellar sky density of 2.8 stars per square degree. Out of the $10^{5}$ stars in the catalog, this implies that only 100 stars would be lensed by a $10^{17} \mathrm{GeV}$ string, which stretched completely across the sky. Even fewer stars will lie in the region of effect at lower energy scales (e.g., of order 1 star at $10^{16} \mathrm{GeV}$ ). While this estimate indicates that our local limits based on tidal effects are not easily superseded by astrometric observations, it also indicates that it might be of interest to analyze the Hipparcos catalog for any indication of local strings.

Another approach to constructing limits may be illustrated by considering the tidal disruption of galaxies. It has been found that roughly $10 \%$ of all observed galaxies exhibit evidence of tidal disruption [20]. Most of these tidal events are clearly due to galaxy-galaxy interactions; at most perhaps $10 \%$ of these events (or $1 \%$ of all galaxies) could possibly be disrupted due to close passage of a global string. If one insists that global strings be separated sufficiently so that at most $1 \%$ of all galaxies will pass within a distance $d$, given 
by Eq. (42), of a string, then that implies that the minimum separation of global strings today is

$$
s \gtrsim 20 d \approx 3\left(\frac{\eta}{10^{16} \mathrm{GeV}}\right) \mathrm{Mpc} .
$$

While the constraint in Eq. (46) is weaker than the cosmological density limit of Eq. (17), it is considerably more ro- bust, since it is based on direct astronomical observations, and is independent of assumptions about cosmological models and the global distribution of strings.

\section{ACKNOWLEDGMENTS}

The work of W.A.H. was supported in part by National Science Foundation Grant No. PHY-9511794.
[1] A. Vilenkin, Phys. Rep. 121, 263 (1985).

[2] A. Vilenkin and A. E. Everett, Phys. Rev. Lett. 48, 1867 (1982).

[3] B. Allen, R. R. Caldwell, S. Dodelson, L. Knox, E. P. S. Shellard, and A. Stebbins, astro-ph/9704160, 1997.

[4] U. Pen, U. Seljak, and N. Turok, astro-ph/9704165, 1997.

[5] J. P. Preskill, Phys. Rev. Lett. 43, 1365 (1979).

[6] R. Gregory, Phys. Rev. Lett. 59, 740 (1987).

[7] J. R. Gott, Astrophys. J. 288, 422 (1985).

[8] W. A. Hiscock, Phys. Rev. D 31, 3288 (1985).

[9] J. Ipser and P. Sikivie, Phys. Rev. D 30, 712 (1984).

[10] M. Barriola and A. Vilenkin, Phys. Rev. Lett. 63, 341 (1989).

[11] A. G. Cohen and D. B. Kaplan, Phys. Lett. B 215, 67 (1988).

[12] G. W. Gibbons, M. E. Ortiz, and F. R. Ruiz, Phys. Rev. D 39, 1546 (1989).

[13] W. A. Hiscock, Phys. Rev. Lett. 64, 344 (1990).
[14] A. Vilenkin and E. P. S. Shellard, Cosmic Strings and Other Topological Defects (Cambridge University Press, Cambridge, United Kingdom, 1994).

[15] U. Pen, D. N. Spergel, and N. Turok, Phys. Rev. D 49, 692 (1994).

[16] C. W. Misner, K. S. Thorne, and J. A. Wheeler, Gravitation (Freeman, San Francisco, 1973).

[17] D. Harari and P. Sikivie, Phys. Rev. D 37, 3438 (1988).

[18] K. Nordtvedt, in Proceedings of the Seventh Marcel Grossmann Meeting on General Relativity, Stanford, California, 1994, edited by R. T. Jantzen et al. (World Scientific, Singapore, 1996), p. 119.

[19] M. Aryal and A. E. Everett, Phys. Rev. D 33, 333 (1986).

[20] B. A. Vorontsov-Velyaminov, Astron. Astrophys. Suppl. Ser. 28, 1 (1977). 